\title{
No-insulation High Temperature Superconductor Winding Technique for Electrical Aircraft Propulsion
}

Yawei Wang, Fangjing Weng, Jianwei Li, Shengnan Zou, Ján Šouc, Fedor Gömöry, Min Zhang, Weijia Yuan

\begin{abstract}
High temperature superconductor (HTS) machine is promising candidate for the electrical aircraft propulsion, due to its great advantage on high power density. However, the HTS machine always suffers the problem of low thermal stability during quench. In this study, we apply a no-insulation (NI) coil technique on the rotor windings of HTS machines to enhance the stability and safety of the electrical aircraft. The NI HTS rotor windings experience ripple magnetic fields, which leads to induced eddy currents through the turn-to-turn contacts. This induced current and accompanying losses will considerably affect the practicality of this technique. To study this issue, an equivalent circuit network model is developed, and it is validated by experiments. Then analysis using this model show that most of induced current flows in the outer turns of the NI HTS coil because of skin effect, and lower turn-to-turn resistivity leads to higher transport current induced and more significant accumulation of turn-to-turn loss. A grading turn-to-turn resistivity is proposed to reduce the transport current induced and AC loss accumulation and meanwhile keep the high thermal stability of the NI HTS coil. Optimization of turn-to-turn resistivity is required when the NI HTS coil is applied in the machines environments.
\end{abstract}

Keywords: superconducting machine, no-insulation coil, AC loss, ripple magnetic fields. 


\section{INTRODUCTION}

The aerospace sector is actively pursuing revolutionary design concepts toward electric aircraft to further reduce the environmental impact of air travel[1-3]. Aggressive targets have been set by both the EU and US to cut aviation emissions. The United Nations' International Civil Aviation Organization plans to cut carbon emissions from airplanes by more than 650 million tons between 2020 and 2040. The EU plans to have greater than $75 \%$ reduction in $\mathrm{CO} 2$ and NOx emissions by 2050[4, 5]. These regulations are the key drivers for aviation manufacturers to design more efficient electric aircraft. The advancement of electric power to improve automobiles serves as the base technology being applied toward aircraft. However, electric aircraft propulsion requires generator \& motor with very high power density, which cannot be achieved by conventional electric machines using copper wires[6-9]. The latest second generation high temperature superconductor (2G HTS) (RE)Ba2Cu3Ox (REBCO) offers a transformative opportunity to develop electrical machines with high power densities, because their current-carrying capability is more than twenty times that of copper. HTS machines have been developed for wind generator, ship propulsion and energy storage successfully, which show great advantages on high power density, compact structure and lightweight, thus is a promising candidate for future electrical aircraft propulsion [6-11].

The aviation propulsion has a very high demand on safety and stability. However, the superconducting machines using HTS coils have always suffered the problem of low thermal stability and quench damages [12-14]. Quench has a significant influence on the safety and reliability of HTS machines, which has to be solved. Conventional quench detection and protection methods applied on low temperature superconductor (LTS) is not suitable for REBCO coils because of its low quench propagation [14-18]. No effective quench detection and protection technique are developed so far for HTS coils. Then, an alternative solution, novel no-insulation (NI) coil technique, was proposed to enhance the thermal stability of REBCO coils in high field magnet [19-21]. The key idea of NI coil is to remove the turn-to-turn electrical insulation of traditional insulated coils. Transport current can bypass the local hot spot through turn-to-turn metallic contacts, so that the quench propagation is prevented and an enhanced thermal stability is achieved, which has been validated by both experiments and simulations [22-26]. Another advantage of the NI HTS coil is the enhanced power density since the elimination of turn-to-turn insulation increase the current density as well as the power density of the HTS machine. Since the NI HTS technique has been successfully applied 
on high field magnets so far [27], there is a great potential that it can also be used in HTS motors/generators to improve the thermal stability and prevent quench damage [28]. Both the EU and NASA have proposed to develop NI HTS machines concept for electrical aircraft propulsion.

HTS motors/generators prefer the synchronous half-superconducting design to minimize the AC losses, in which the HTS is only applied on rotors windings and the stator windings still use copper wires. The application of HTS windings on rotor can significantly increase the magnetic field at air gap, so that the power density of the machine is increased. In synchronous machines, rotation speed of fundamental fields generated by stator windings is same with the rotation speed of rotor, thus the HTS rotor windings serve as a DC magnet during operations [29-31]. Besides the fundamental fields, the ripple fields from stators are not synchronous to the rotor windings. Therefore, the HTS coils on the rotors of machines will suffer AC ripple magnetic fields. AC loss of HTS coils induced by the ripple fields has always been a critical issue in the design of conventional HTS machines using insulated HTS coils, since it has a considerable influence on the efficiency of HTS machines[32]. If NI HTS coils was applied on rotors of machines, the ripple field may have a considerable influence on the electromagnetic behavior of NI HTS coils due to the absence of turn-to-turn insulation, like induced eddy current and extra loss on turn-to-turn contacts[33, 34]. This has never been studied thoroughly, which may a great challenge for the thermal stability and efficiency of HTS machines with NI technique.

This work aims to investigate the influence of ripple magnetic fields in machines on the electromagnetic behavior of NI HTS NI coils numerically and experimentally, in order to investigate the practicability of NI HTS machine design. Since the current can flow along any direction in the NI HTS coils, an equivalent circuit modeling method is developed to calculate the current and loss of NI HTS coils exposed in AC ripple background magnetic field. A NI HTS coil is wound b REBCO tapes for test, and a copper solenoid is wound to generate the background ripple magnetic field. Then, the NI HTS coil is exposed in the ripple magnetic field and its electromagnetic behavior is tested. Results from simulation and experiments are compared to validate the model. Then the distribution of induced eddy current and $\mathrm{AC}$ loss is analyzed using this model, and the influence of turn-to-turn resistivity, field frequency and amplitude on the eddy current are also discussed. 


\section{Numerical Model}

The 2G HTS REBCO tape is a coated conductor with high aspect, as shown in Figure 1(a) and 1(b). It has multiple layers, and the superconducting layer is covered by metallic layers (copper, silver and hastelloy), as shown in Figure 1 (b). The NI HTS coil is often wound with pancake structure, as shown in Figure 2(a). When the NI coil is operated in DC environment below critical current, all the current flows in the superconducting layer along azimuthal current, since the resistivity of superconducting layer is much lower than the metallic layer and the metallic layers serve as in insulation. When the NI HTS coil is exposed to AC ripple magnetic fields, eddy current can be induced in the coil, and some current can flow along the radial direction through the turn-to-turn contacts[35, 36].

To calculate the distribution of this eddy current in NI HTS coils, an equivalent circuit model is developed to study the distribution of the induced eddy current as well as turn-to-turn loss in the NI HTS coil. In this model, the current is decomposed to the azimuthal component $i$ and radial component $j$. Each turn of the coil is subdivided to fine arc element, and each element is equivalent to lumped circuit model. The whole coil is equivalent to a distributed circuit network [37-41], as shown in Fig. 2(c). Each dependent current node has four current branches, two azimuthal branches and tow radial branches, in which the governing equation can be derived from Kirchhoff current law. Each dependent circuit mesh also has four current braches, two radial branches and two azimuthal branches, in which the governing equation can be derived from Kirchhoff voltage law. The two radial branches represents the resistive voltage generated on turn-to-turn contacts. Therefore, the governing equation of this network model can be expressed as:

$$
\left\{\begin{array}{l}
\sum_{k} i_{k}+\sum_{k} j_{k}=0 \\
u_{k}-u_{k+n_{e}}-j_{k-1} \frac{\rho_{r}}{S_{r, k-1}}+j_{k} \frac{\rho_{r}}{S_{r, k}}=0
\end{array}\right.
$$

where $i_{k}$ and $j_{k}$ are the azimuthal and radial current respectively. $S_{r, k}$ is the radial surface of arc element, $\rho_{r}$ is the equivalent radial resistivity with a unit of $\mu \Omega \cdot \mathrm{cm}^{2}$ [42], which is obtained in the following measurement. $u_{k}$ is the voltage of the $k$-th azimuthal branch, including the resistive voltage and inductive voltage. 
The background magnetic field can be generated by equivalent virtual coils in this network model, as shown in Fig. 2(b). then the influence of the background field can be represented by coupling through the mutual inductance $M_{e}$, as shown in Fig. 2(c). Therefore, the inductive voltage of $u_{k}$ has two parts: one is from the coil itself, the other one is from the virtual external coil:

$$
u_{k}=\sum M_{k, l} \frac{d i_{l}}{d t}+M_{e k} \frac{d I_{e}}{d t}+V_{R, k}\left(i_{k}, I_{c}\right)
$$

Where $M_{k, l}$ is the multiple inductance between two arc element in the NI HTS coil, $M_{e k}$ is the multiple inductance between $k$-th arc element and the external equivalent virtual coils. Ie is the transport current of the equivalent virtual coil, the amplitude and frequency of the background field can be changed by adjusting the amplitude and frequency of this current. $V_{R, k}$ is the resistive voltage of the $k$-th azimuthal circuit branch. As shown in Fig. 1(c), the resistance of HTS tape consists of two parallel resistances: resistance of superconducting layer $R_{s c}$ and resistance of metallic layer $R_{n}$. The resistance of superconductor is nearly zero below critical current Ic, and it increase dramatically when the current is higher than critical current. It can be obtained directly from the $E-J$ power law of REBCO conductor:

$$
E=E_{0}\left(\frac{J}{J_{c}}\right)^{n}
$$

where $J_{c}$ is the critical current density, $E_{0}=1 \mu \mathrm{V} / \mathrm{cm}, n=25 \sim 35$. Then the distribution of current induced by ripple magnetic field can be calculated by solving this numerical model.

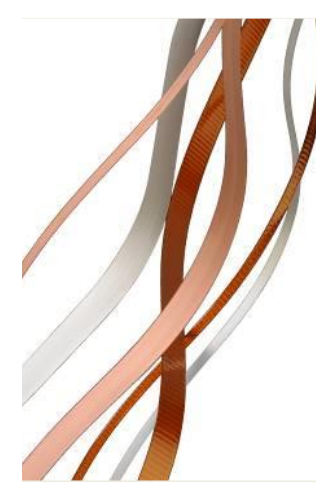

(a) Photo of ReBCO tape

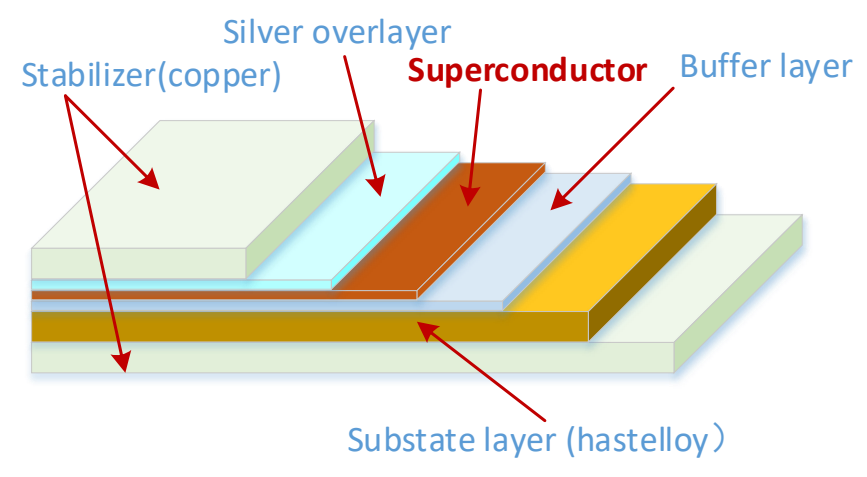

(b) Layer structure of ReBCO tape

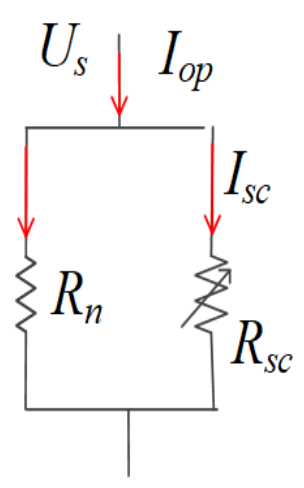

(c) Circuit model for ReBCO tape

Fig. 1. (a) Photo of 2G HTS ReBCO tape; (b) The multiple layer structure of the 2 G HTS tape; (b) The equivalent circuit model of ReBCO tape 


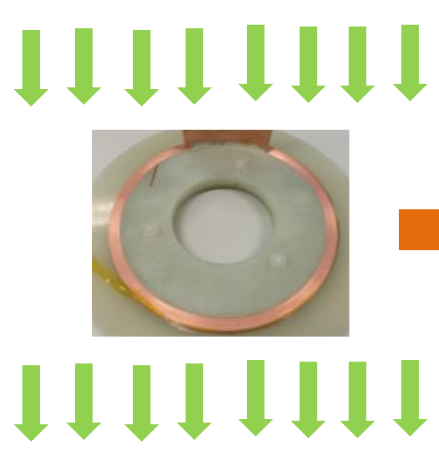

(a) NI HTS coil exposed to ripple magnetic field

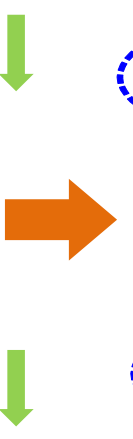

(b) NI HTS coil with virtual external coil

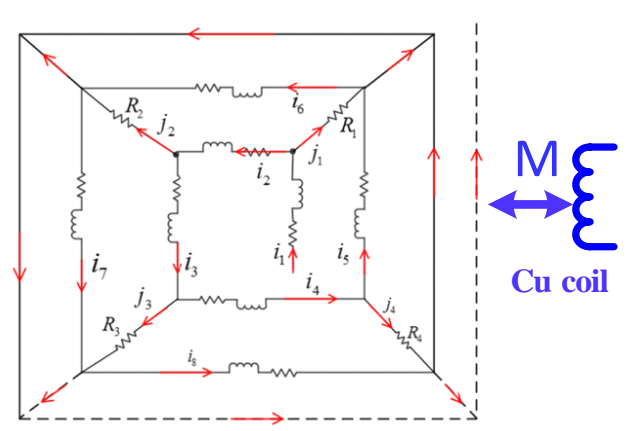

(c) Equivalent circuit network model

Fig. 2. (a) NI HTS pancake coil exposed to background ripple fields; (b) The schematic illustration of NI HTS coil with virtual coils generating the background ripple fields; (c) The schematic illustration of the equivalent circuit network model for NI HTS coils exposed to background fields. Notice that each turn is subdivided to 4 elements in this figure, which is for clear presentation.

\section{EXPERIMENTS AND MODEL VALIDATION}

\section{A. Experimental setup}

To validate the above model, A NI HTS coil is wound by REBCO tapes from SuNAM, South Korea, as shown in Fig. 3(a). The tape has a width of $4 \mathrm{~mm}$ and thickness of $0.25 \mathrm{~mm}$. Its critical current at $77 \mathrm{~K}$ is $190 \mathrm{~A}$ and the coil's critical current is $130 \mathrm{~A}$. The NI coil has a single pancake (SP) geometry, and two copper sheets are used as current leads for fast discharge test. More details of the coil is shown in the Table 1. A solenoid copper coil is wound to generate the background magnetic field, as shown in Fig. 3(b). It is energized by an AC power supply. Specification of this solenoid is shown in the Table 1. As shown in Fig. 4, the NI HTS coil is placed at the central position of the copper solenoid, thus the NI HTS coil is exposed to an AC ripple magnetic field generated by the copper coil. A hall sensor is placed at the centre of the HTS coil to measure the magnetic field. 


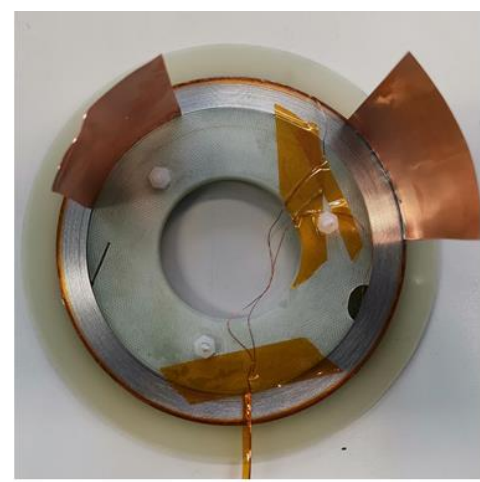

NI HTS coil

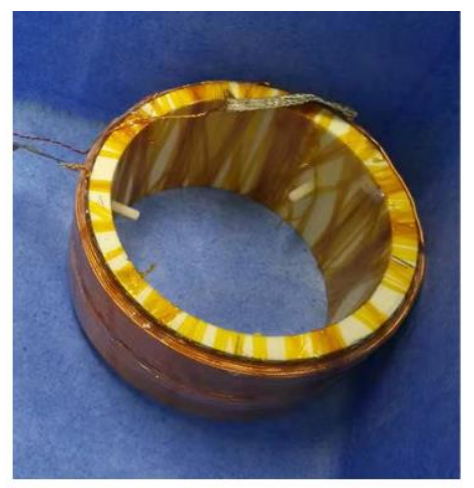

Copper coil

Fig. 3. Photos of test NI HTS coil and copper solenoid coil.

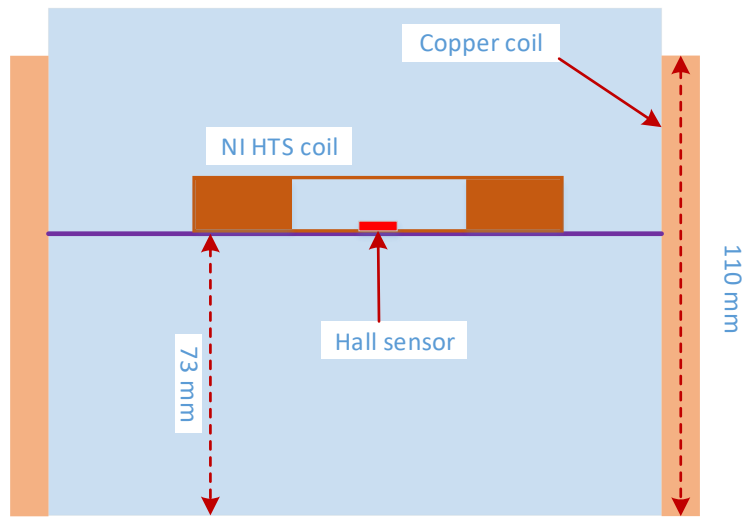

Fig. 4. Location of the NI HTS coil and copper coil during test.

TABLE I.

SPECIFICATION OF THE TEST HTS COILS AND COPPER COIL

\begin{tabular}{lll}
\hline \hline Parameters & NI HTS coil & Copper coil \\
\hline Coil type & Single pancake & Solenoid \\
Inner diameter & $100 \mathrm{~mm}$ & $215 \mathrm{~mm}$ \\
Outer diameter & $121 \mathrm{~mm}$ & $235 \mathrm{~mm}$ \\
Height & $4.0 \mathrm{~mm}$ & $110 \mathrm{~mm}$ \\
Number of turns & 45 & 289 \\
Critical current, @77 K & $130 \mathrm{~A}$ & $/$ \\
Inductance & $406 \mu \mathrm{H}$ & $18.7 \mathrm{mH}$ \\
Filed per Ampere & $506 \mu \mathrm{T} / \mathrm{A}$ & $1.42 \mathrm{mT} / \mathrm{A}$ \\
Tape producer & $\mathrm{SuNAM}$ & $/$ \\
Width/thickness of tape & $4.0 / 0.25 \mathrm{~mm}$ & $/$ \\
Turn-to-turn resistivity & $96.7 \mu \Omega \cdot \mathrm{cm}^{2}$ & $/$ \\
\hline \hline
\end{tabular}

\section{B. Fast discharge test}

Turn-to-turn resistivity $\rho_{r}$ is a key parameter of NI HTS coil, which distinguishes it from conventional insulated HTS coils. It is also a necessary for the simulation of current distribution in the NI coil, as shown in Eq. (1). Both 
the HTS coil and copper coil is immersed in liquid nitrogen during the test. Firstly, fast discharge test are performed on the NI HTS coils to obtain the turn-to-turn resistivity. During the fast discharge test, the copper solenoid coils is in open circuit, the NI coil is first ramped to a transport current below critical current and kept at this current for the time long enough to eliminate the charging delay. Then the current is switched off by an air circuit breaker, and all the magnetic energy stored in the coil is dissipated in the coil itself. The coil voltage is measured in the process, and its decay is shown in Fig. 5. Here the initial stable transport current of NI coil A is 40 A, 80 A and 100 A respectively. The field decay matches the following equation [19]:

$$
U=U_{0} e^{-\frac{t}{L / R_{c}}}
$$

where $U$ is the coil voltage measured, $B_{0}$ is the initial coil voltage, $L$ is inductance of the coil, $R_{c}$ is the equivalent radial resistance. The time constant can be obtained directly from the results in Fig. 5. The equivalent turn-to-turn resistivity $\rho_{t}$ with a unit of $\mu \Omega \cdot \mathrm{cm}^{2}$ can be calculated by[43]:

$$
\rho_{t}=R_{c} / \sum_{k=1}^{N_{t}} \frac{1}{2 \pi r_{k} w_{d}}
$$

where $N_{t}$ is the total number of turns, $r_{k}$ is the radius of the $k$-th turn, $w_{d}$ is the width of the tape. The equivalent turnto-turn resistivity of this NI HTS coil is $96.7 \mu \Omega \cdot \mathrm{cm}^{2}$.

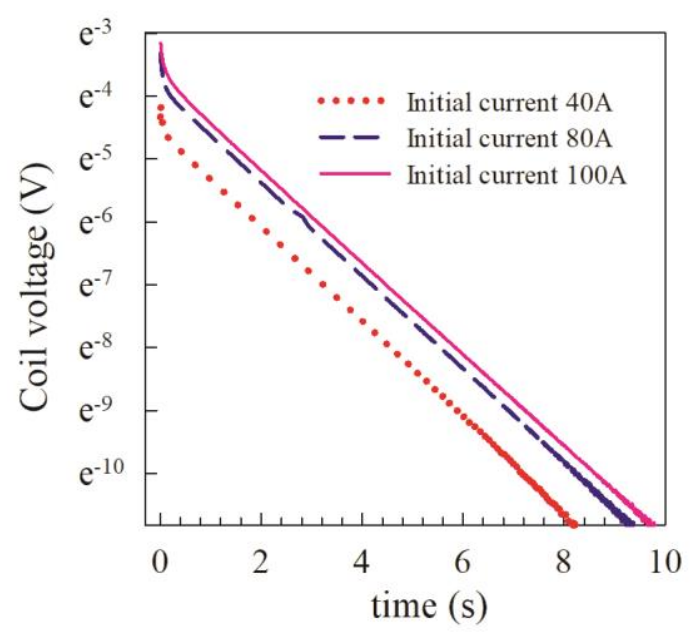

Fig. 5. The decay of the NI HTS coil's terminal voltage during the fast discharging test. 


\section{Model validation}

As shown in Fig. 4, the magnetic field at coil centre should be lower than the background field generated by the copper coil, since the induced eddy current in the NI HTS coil reduces the central magnetic field. Therefore, we can validate the numerical model by comparing the central magnetic fields from measurements and simulations. The NI HTS coils do not carry a transport current, and they are open circuited during the measurement. The copper leads are removed before this measurement to eliminate the influence of eddy current induced in copper leads. Fig. 5 shows the variation of magnetic fields at coil centre when the copper coil is energized by a transport current with an amplitude of $20.66 \mathrm{~A}$ and frequency $40 \mathrm{~Hz}$. Notice that the ripple background field here is that at same position when there is no NI HTS coils. It is used to represent the background field generated by the copper coil, and its amplitude is $29.3 \mathrm{mT}$ in Fig. 6 . Then, we change the amplitude of the background field, and measure the amplitude of the magnetic field at coil centre under different ripple fields, as shown in Fig. 7. The results from the above model show a good agreement with that from measurement. The discrepancy between simulation and experiment have two main possible factors: first, the turn-to-turn resistivity used in simulation is measured from a fast discharge test, and it may change in the following operations, like removing the current leads and the vibration induced by ripple fields and liquid nitrogen boiling. Second, the turn-to-turn resistivity is assumed uniform among turns in the simulation, while it may vary among turns in fact. However, the results are good enough to validate the modelling method.

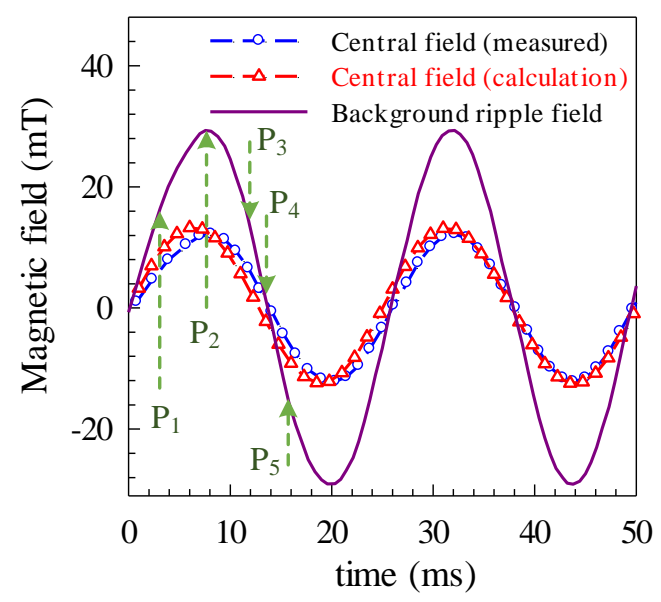

Fig. 6. The measured and calculated magnetic field at the centre of the NI HTS coil when it is exposed to a ripple background magnetic field with a frequency of $40 \mathrm{~Hz}$. 


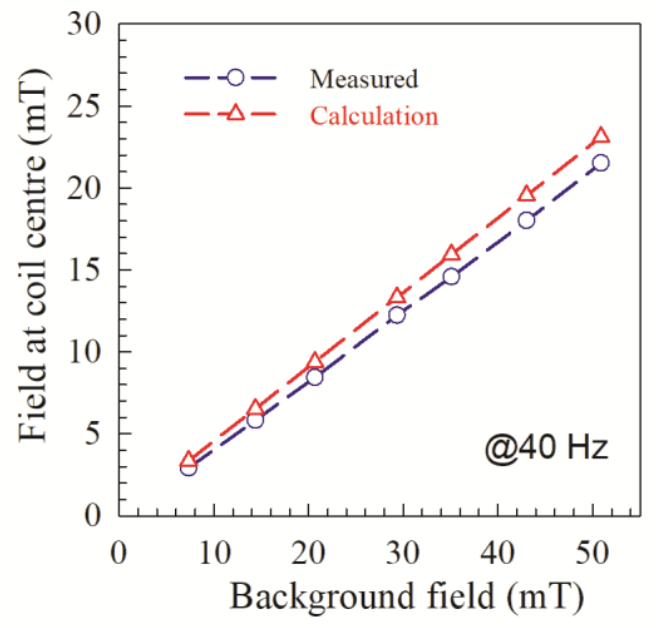

Fig. 7. The amplitude of magnetic field at the NI HTS centre under different ripple background magnetic fields, their frequency is $40 \mathrm{~Hz}$. 

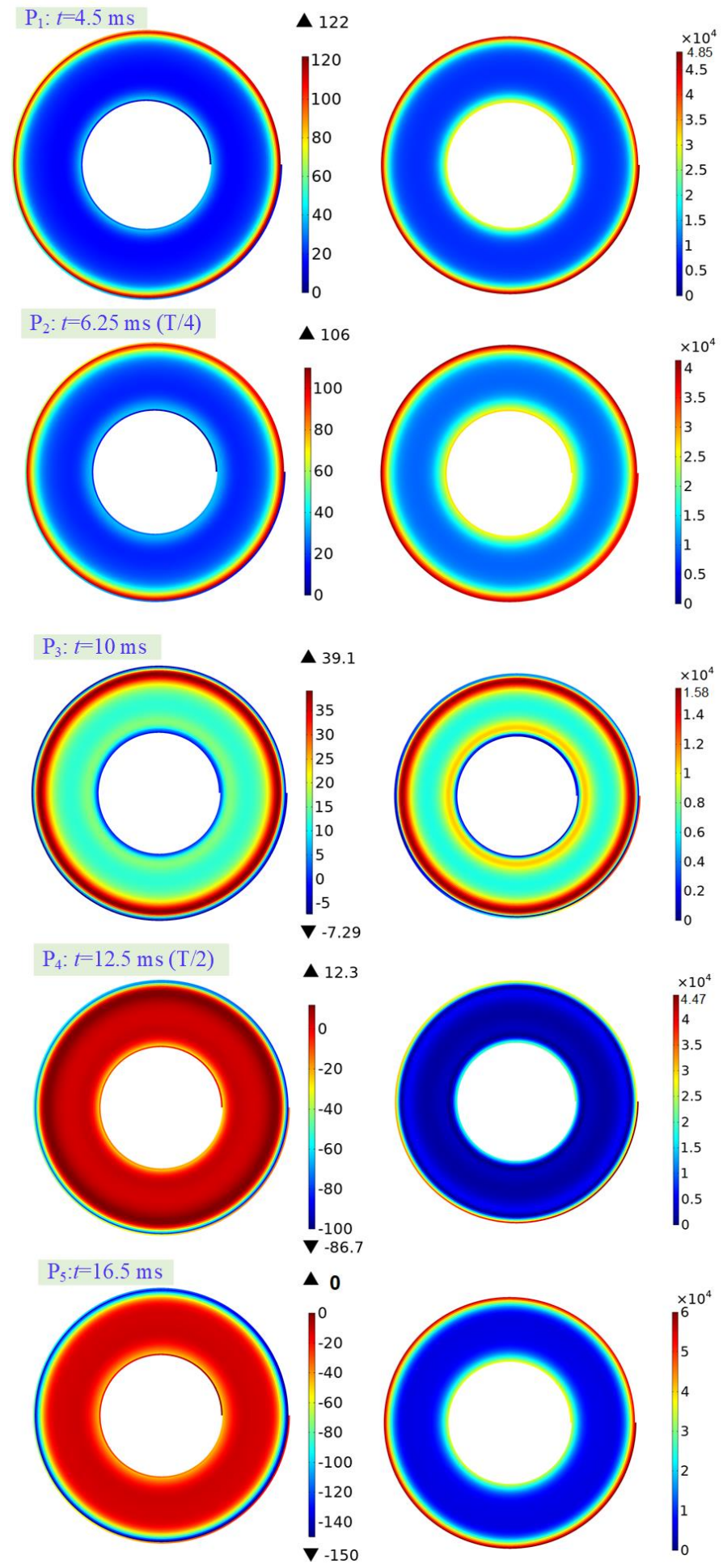

(a) azimuthal current (A)

(b) radial current density $\left(\mathrm{A} / \mathrm{m}^{2}\right)$

Fig. 8. The distribution of induce eddy current (azimuthal current and radial current) in the NI HTS coil when it is exposed to ripple background field generated by copper solenoid coil as shown in Fig. 5; the background field is $29 \mathrm{mT} / 40 \mathrm{~Hz}$, the turn-toturn resistivity of the NI HTS coil is $96.7 \mu \Omega \cdot \mathrm{cm}^{2}$. The thickness of each turn is enlarged 5 times for a better presentation. 


\section{RESUlTS AND DiscuSSION}

\section{A. Induced eddy current and losses}

The distribution of induce eddy current is analysed using the above model developed, as shown in Fig. 8. Here 5 moments $\mathrm{P}_{1}, \mathrm{P}_{2}, \mathrm{P}_{3}, \mathrm{P}_{4}, \mathrm{P}_{5}$ is selected during a cycle, as shown in Fig. 6. The azimuthal current, with a unit of $\mathrm{A}$, flows in superconducting layer, and its positive direction is anticlockwise direction, as shown in Fig. 8(a). The radial current follows through the turn-to-turn contacts, Fig. 8(b) shows the normal value of the radial current density, whose unit is $\mathrm{A} / \mathrm{m}^{2}$ here. We observe kind of skin effect, where most of eddy current is generated on the inner and outer turns of the NI HTS coil, especially the outer turns. That's because the outer turns have relatively larger diameter, thus couples more external magnetic flux than other turns. Then the induced eddy current has a field shielding effects on the other turns, so that less current is induced on these turns. The azimuthal current (transport current induced) flows and the closed circuit is formed through the turn-to-turn contacts, which leads the radial current. Thus, the distribution of radial current is almost same with that of azimuthal current. The induced currents show a uniform distribution along the angular direction of the coil, but the current on the outermost turn shows a considerable non-uniform distribution along angular direction. The zone near the current lead has higher current than other zones on the same turn.

The maximum transport current induced (azimuthal current) is a critical issue for the HTS coil, since the quench risk increases rapidly with the transport current. The transport current in HTS tape has to be below critical current during operations. The results in Fig. 8 also show that the azimuthal current is not synchronous with the amplitude of the background field. The maximum azimuthal current induced does not occurs at the peak point $\left(\mathrm{P}_{2}\right)$ of the background fields, but before these moments, $\mathrm{P}_{1}$ and $\mathrm{P}_{5}$. That's because the induced voltage is generated by variation of background fields, and it has the highest rate of change at these moments. Therefore, $\mathrm{P}_{1}$ and $\mathrm{P}_{5}$ is the weak moment with higher quench risk for the NI HTS coil. Fig. 9 shows the azimuthal current induced by higher background fields, and an overcurrent (>190 A) is observed on the outermost turn of the NI HTS coil when the background field is high enough. The penetration depth of induced current increases rapidly with the background field when overcurrent is induced. This is because that the resistance of HTS increases dramatically above the critical current, which forces more current to other turns. 


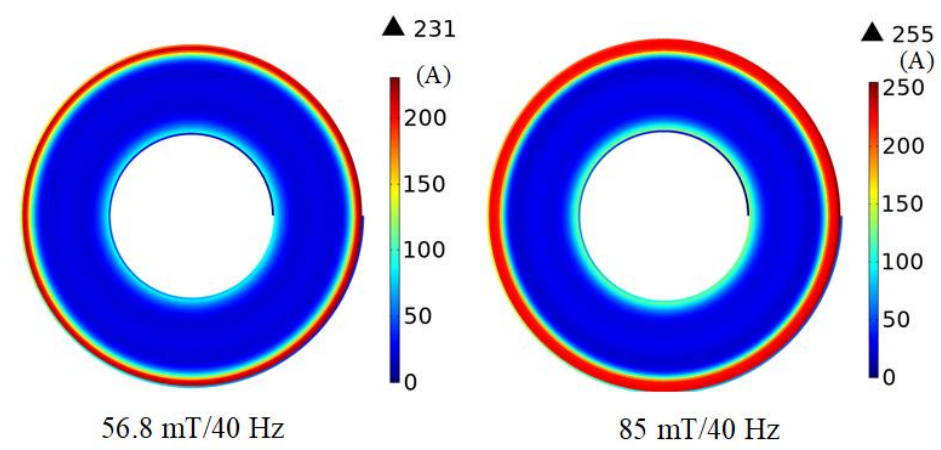

Fig. 9. The distribution of induced azimuthal current in the NI HTS coil, when it is exposed in higher background fields, $56.8 \mathrm{mT} / 40 \mathrm{~Hz}$ and $85 \mathrm{mT} / 40 \mathrm{~Hz}$; the turn-to-turn resistivity of the NI HTS coil is $96.7 \mu \Omega \cdot \mathrm{cm}^{2}$.

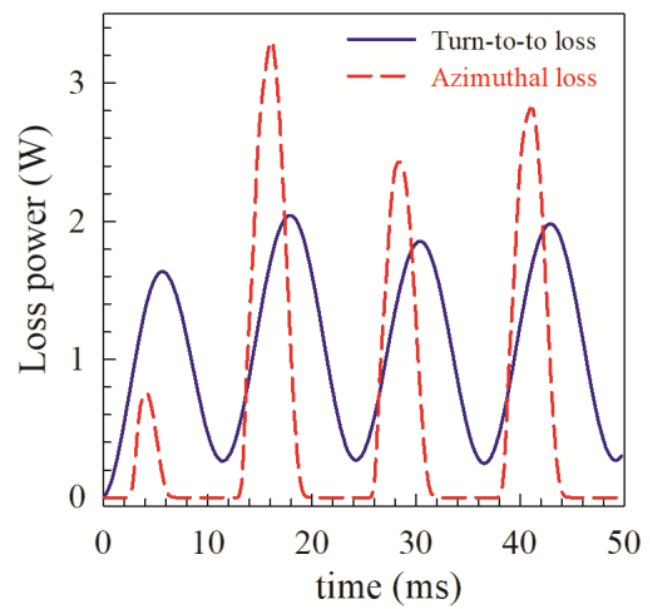

Fig. 10. The losses generated by radial current (turn-to-turn loss) and azimuthal current when the NI HTS coil is in background field is $56.8 \mathrm{mT} / 40 \mathrm{~Hz}$, the turn-to-turn resistivity of the NI HTS coil is $96.7 \mu \Omega \cdot \mathrm{cm}^{2}$.

Losses are generated by the eddy current induced, which consist of two parts: the radial current induced generates a resistive loss on the turn-to-turn contacts, which is called 'turn-to-turn loss' in this study; the losses generated by transport (azimuthal) current is called 'azimuthal loss'. The azimuthal current flows in superconducting layers below critical current, and a magnetization loss is generated in superconductors by this $\mathrm{AC}$ transport current[33, 34]. When the induced transport (azimuthal) current is over the critical current, the resistance of superconducting layer increases dramatically, some current is forced out to metallic layers, and thus a huge resistive loss is generated by this overcurrent. Fig.10 shows the turn-to-turn loss and azimuthal loss induced by background fields $51 \mathrm{mT} / 40 \mathrm{~Hz}$. The peak moment of radial loss matches well with the peak moment of azimuthal current in Fig.8. The induced 
losses should have same distribution on the NI HTS coil with the induced current, most of the eddy loss accumulated on the outermost turns of the NI HTS coil.

The above analysis show that both the eddy current and losses accumulate on the outermost turns of the NI HTS coil, therefore, the outmost turns have a much higher quench risk than other turns, and a special design is required on this zone during electromagnetic and cooling design.

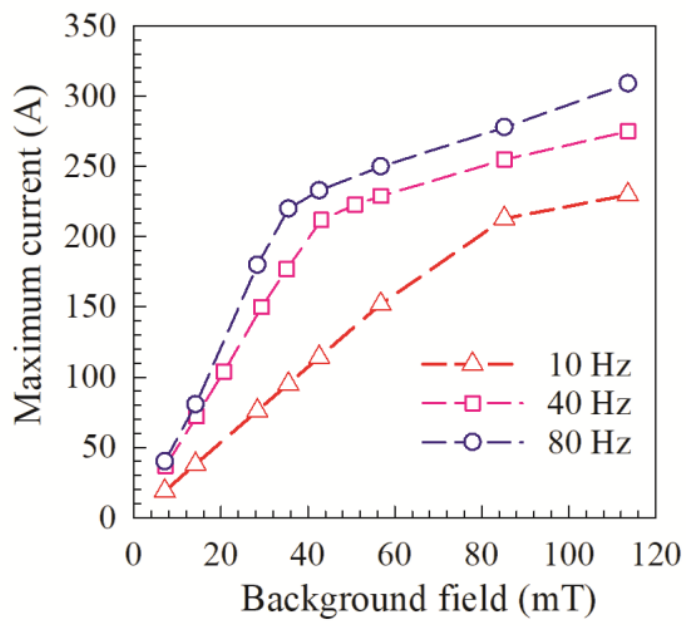

Fig. 11. The dependence of maximum azimuthal current on the amplitude of background field, the turn-to-turn resistivity of the NI HTS coil is $96.7 \mu \Omega \cdot \mathrm{cm}^{2}$.
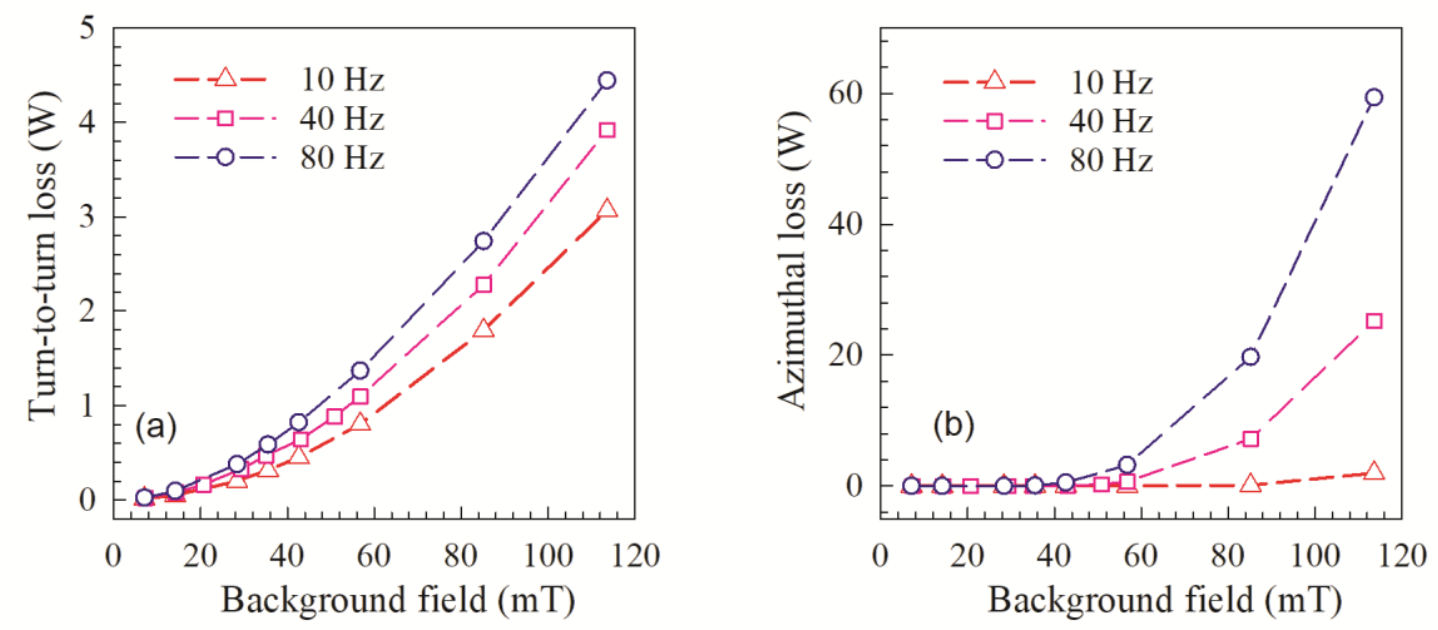

Fig. 12. The dependence of turn-to-turn loss (Figure a) and azimuthal loss (Figure b) on the amplitude of background field, the turn-to-turn resistivity of the NI HTS coil is $96.7 \mu \Omega \cdot \mathrm{cm}^{2}$. 


\section{B. Influence of background fields}

Fig. 11 shows the dependence of the maximum azimuthal current induced (transport current in HTS tape) on the amplitude of the background field. The maximum induced current increases almost linearly with the amplitude of the background fields, when it is below critical current (190 A). Above the critical current, it shows a very slowly increase with the further increase of background fields. Below critical current, the azimuthal resistance of HTS is nearly zero and the turn-to-turn resistance is constant, thus the induced current is almost proportional to the amplitude of the background field. Above the critical current, the resistance of HTS increases rapidly, the maximum transport (azimuthal) current is limited, and thus the increasing rate drops fast.

Fig.12 shows the dependence of eddy loss on the amplitude of background fields. The turn-to-turn loss increases rapidly with the amplitude of background fields, and the increasing rate is a little higher than that of linear increase. The azimuthal loss is nearly zero when the induced transport (azimuthal) current is below critical current, which is much lower than the turn-to-turn loss. When an overcurrent is induced, the azimuthal loss increases dramatically with the further increase of background fields, which can be one magnitude of order higher than turn-to-turn loss. Therefore, a special design is required to prevent this induced overcurrent. Below the critical current, the azimuthal loss is ignorable compared to the turn-to-turn loss.

Fig. 13 shows the influence of field frequency on the distribution of induced transport current. Higher frequency leads to more significant non-uniform current distribution on the NI HTS coil, more induced transport current accumulated on the outermost turns, due to the skin effect. Fig. 14 shows that the maximum induced transport current increases continually with the frequency of background fields. The results show that a very small AC field can induce a very high transport current in the NI HTS coil, especially at high frequency. As shown in Fig. 14, a background field $14 \mathrm{mT} / 200 \mathrm{~Hz}$ can leads to a transport current up to $110 \mathrm{~A}$ on the outermost turn of the NI HTS coil, while the critical current of HTS tapes is only 190 A. In the HTS machine environment of electrical aircraft, the frequency of ripple field can be much higher than $200 \mathrm{~Hz}$, and the rated current of HTS coil is often more than $60 \%$ of the critical current. Fig. 15 shows the dependence of the turn-to-turn loss power on field frequency. The logarithm of turn-to-turn loss power is almost proportional to the logarithm of field frequency. The turn-to-turn loss power increases rapidly with field frequency when the frequency is in a low range, but the growth rate drops 
continually with field frequency. Therefore, the ripple background field with higher frequency leads to more significant eddy current accumulation, higher transport current induced and more eddy loss, which can considerably increase the quench risk of the NI HTS coil during operations.
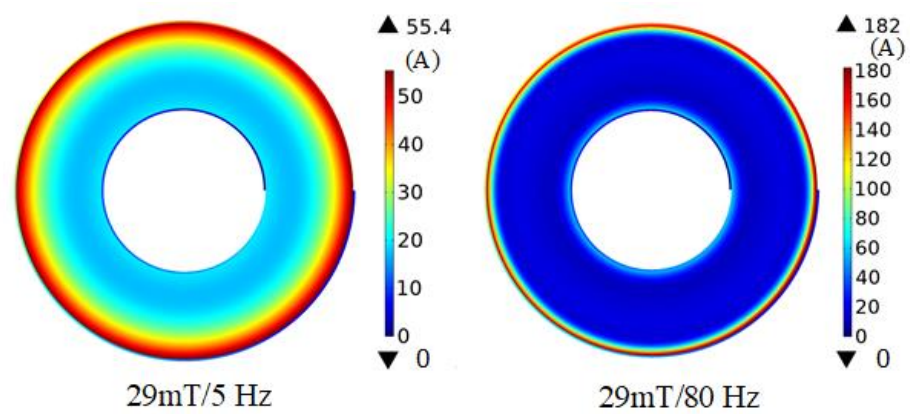

Fig. 13. The distribution of induced transport (azimuthal) current in the NI HTS coil, the background fields are $29 \mathrm{mT} / 5 \mathrm{~Hz}$ and $29 \mathrm{mT} / 80 \mathrm{~Hz}$, the turn-to-turn resistivity of the NI HTS coil is $96.7 \mu \Omega \cdot \mathrm{cm}^{2}$.

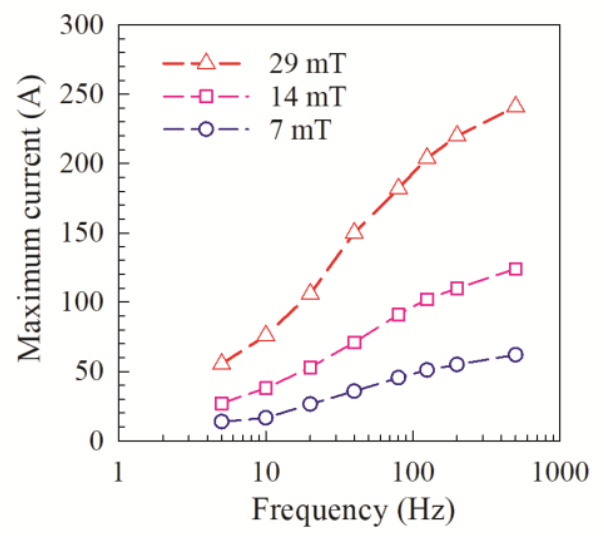

Fig. 14. The dependence of maximum induced transport (azimuthal) current on the frequency of background field, the turn-toturn resistivity of the NI HTS coil is $96.7 \mu \Omega \cdot \mathrm{cm}^{2}$.

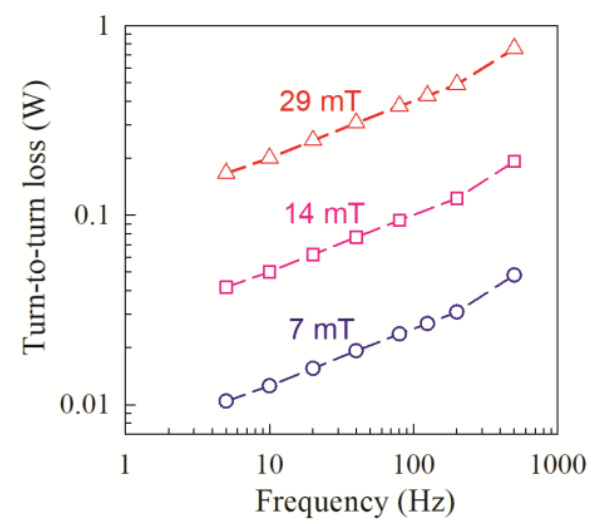

Fig. 15. The dependence of turn-to-turn loss power on the frequency of background field, the turn-to-turn resistivity of the NI HTS coil is $96.7 \mu \Omega \cdot \mathrm{cm}^{2}$. 


\section{Influence of turn-to-turn resistivity}

The turn-to-turn resistivity of NI HTS coils ranges from $1 \mu \Omega \cdot \mathrm{cm}^{2}$ to $10000 \mu \Omega \cdot \mathrm{cm}^{2}$, and it depends on the winding tension, surface condition, materials of substrate and stabilizer [44-46]. We change the turn-to-turn resistivity of the NI coil and re-calculate the distribution of eddy current induced by ripple background fields, as shown in Fig. 16. With lower turn-to-turn resistivity, a more significant skin effect is observed, and a higher transport (azimuthal) current is induced in outermost turns of the NI coil. When the turn-to-turn resistivity is low enough (1 $\mu \Omega \cdot \mathrm{cm}^{2}$ in Fig. 16), the induced current path closes at the connecting position of the outermost turn and its adjacent turns, thus the radial current shows a significant accumulation at this point, which is near the current lead generally, as shown in Fig. 16. This may induce a local hot spot and increases the quench risk to some extent, thus this position is weak point of the NI HTS coil under ripple fields. The radial current shows a uniform distribution along the angular direction when the turn-to-turn resistivity is high enough.

Fig. 17 shows the dependence of the maximum transport (azimuthal) current on the turn-to-turn resistivity. The results show that the maximum transport current induced can be reduced significantly by increasing the turn-to-turn resistivity. If the background field is high enough ( $57 \mathrm{mT}$ for example), increasing turn-to-turn resistivity may lead to a slight increase on the transport current induced, when the turn-to-turn resistivity is in a low range. Then, the transport current induced drops rapidly with the further increase of the turn-to-turn resistivity. Fig.18 shows the dependence of the induced turn-to-turn loss on the turn-to-turn resistivity. The turn-to-turn loss increases with the turn-to-turn resistivity when the resistivity is low. When the resistivity is high enough, the turn-to-turn loss drops rapidly with the further increase of turn-to-turn resistivity. Since the turn-to-turn loss is the product of turn-to-turn contact resistance and the square of induced eddy current, lower turn-to-turn resistivity does not always lead to higher turn-to-turn losses. If the turn-to-turn resistivity drops to zero, the winding of NI HTS coil will be shielded from the external $\mathrm{AC}$ fields, and the $\mathrm{AC}$ loss will be reduced. On the other hand if the turn-to-turn resistivity is infinite, the NI HTS coil will change to an insulated HTS coil, thus only the superconductor magnetization loss is induced, which is much lower than AC loss of NI HTS coils. In other words, the behaviour is typical for eddy current loss in metals, where the decrease of resistivity increases the loss first reaching a peak point, and afterwards causes it to drop again. Obviously, this peak point should be avoided when the NI HTS coil is applied in ripple fields. 
Therefore, the AC loss of NI HTS coils exposed to AC external magnetic fields can be reduced by both increasing and reducing the turn-to-turn resistivity. Lowering turn-to-turn resistivity always means better current redistribution during local quench, which can increase the thermal stability of NI HTS coil; while this will lead to more serious charging delay, which is also challenging for the fast magnetization and demagnetization of NI HTS coils [43, 47]. Lower turn-to-turn resistivity will also leads to higher transport current induced, which will increase the quench risk of the NI coil to some extent. Increasing the turn-to-turn resistivity can significantly reduce the eddy current and turn-to-turn loss induced, while this can prevent current redistribution among turns during a local quench, thus may considerably reduce the thermal stability of NI HTS coils. Therefore, an optimal design on the turn-to-turn resistivity is required on the NI HTS coils when it is applied on machines.
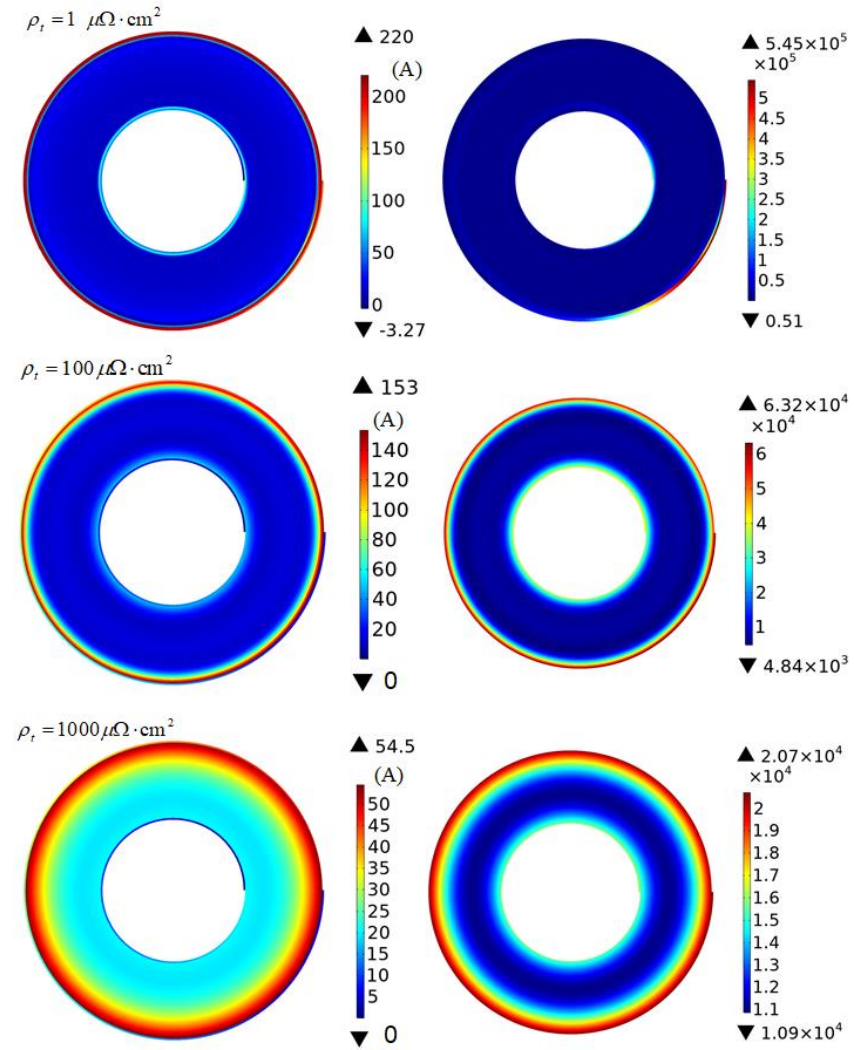

(a) azimuthal current (A)

(b) radial current density $\left(\mathrm{A} / \mathrm{m}^{2}\right)$

Fig. 16. The distribution of induced transport (azimuthal) current and radial current in the NI HTS coils with different turn-toturn resistivity $1 \sim 1000 \mu \Omega \cdot \mathrm{cm}^{2}$, the background field is $29 \mathrm{mT} / 50 \mathrm{~Hz}$. 


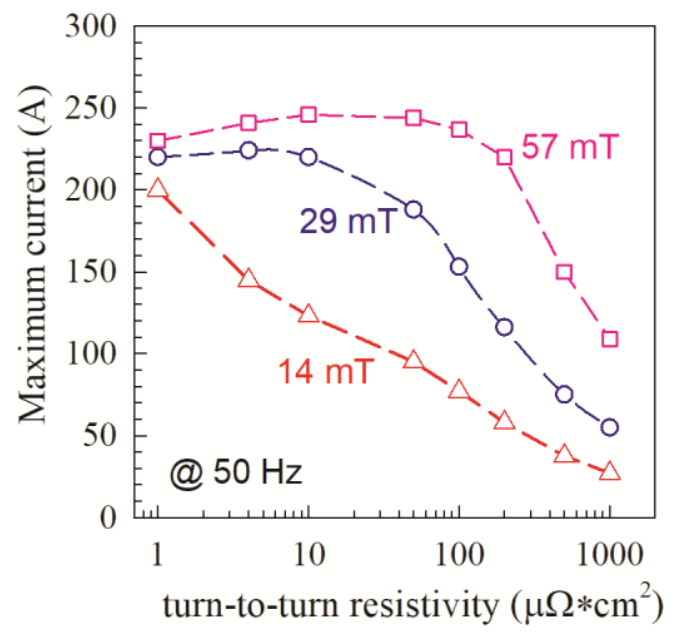

Fig. 17. Dependence of maximum induced transport (azimuthal) current on the turn-to-turn resistivity of the NI HTS coil, the frequency of the background field is $50 \mathrm{~Hz}$.

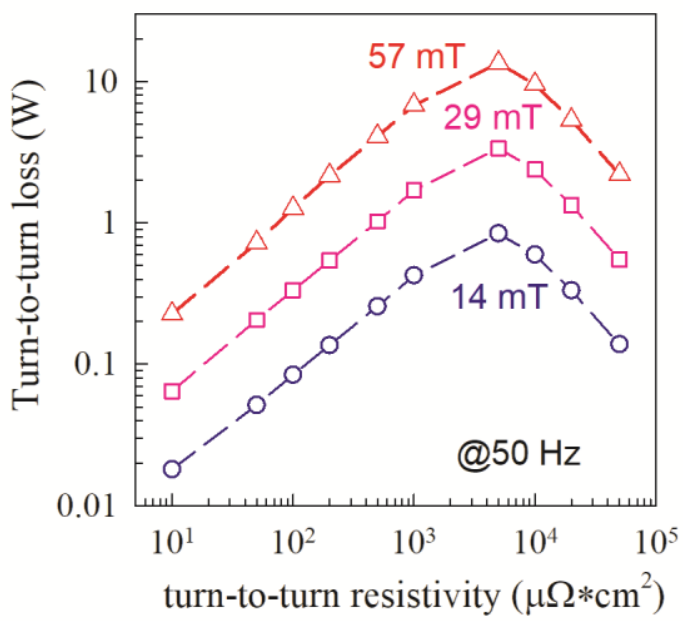

Fig. 18. Dependence of turn-to-turn loss on the turn-to-turn resistivity of the NI HTS coil, the frequency of the background field is $50 \mathrm{~Hz}$.

\section{Grading turn-to-turn resistivity technique}

The above analysis show that the transport current induced can be reduced significantly by increasing the turn-toturn resistivity, while most of induced current accumulate on the outermost turns of the NI HTS coil. All these studies are based on an uniform turn-to-turn resistivity among turns of the NI HTS coil. To reduce the induced transport current and meanwhile keep a better thermal stability of NI coils, a grading turn-to-turn resistivity technique is proposed, in which a higher turn-to-turn resistivity is applied on the turns near outermost zone of the 
NI coil to reduce the current accumulation, a lower turn-to-turn resistivity is applied on the other turns to keep a good thermal stability of the NI coil. A case study is shown in the Fig. 18. The case0 is an uniform turn-to-turn resistivity distribution among turns $100 \mu \Omega \cdot \mathrm{cm}^{2}$, and the distribution of the induced transport(azimuthal) current is shown in the Fig. 15, in which the maximum azimuthal current is 153 A. Fig. 18(a) shows three grading turn-toturn resistivity cases, in which the resistivity of the outermost turns are enlarged. The results show that the current accumulation on the outermost turns are reduced considerably, and hence the turn-to-turn loss. The maximum transport current induced is reduced significantly. Therefore, the grading turn-to-turn resistivity can significantly enhance the thermal stability of the NI HTS coil exposed in ripple magnetic fields.
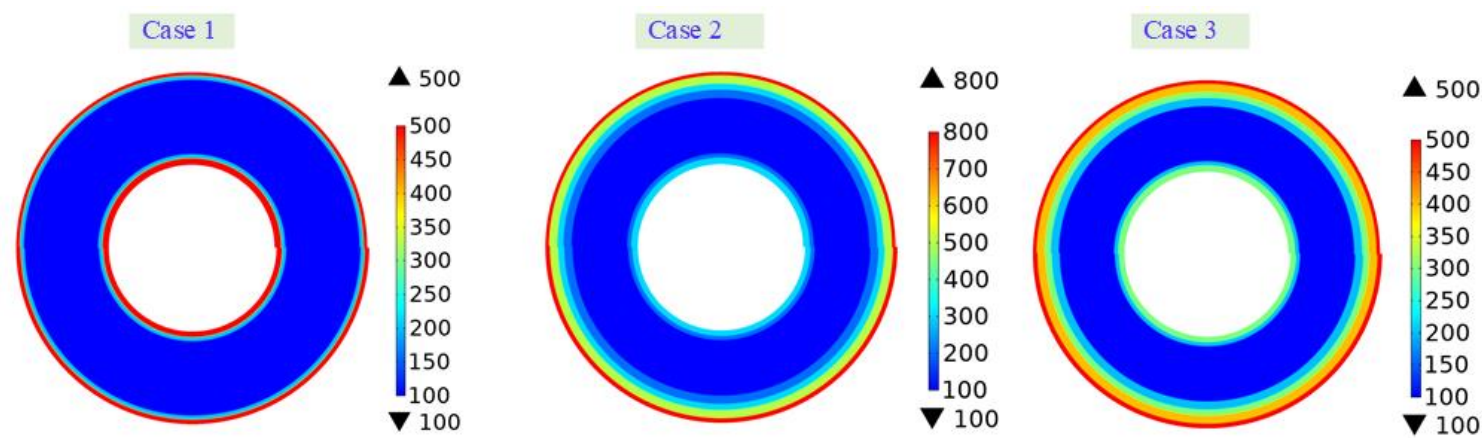

(a) Grading turn-to-turn resistivity $\left(\mu \Omega \cdot \mathrm{cm}^{2}\right)$
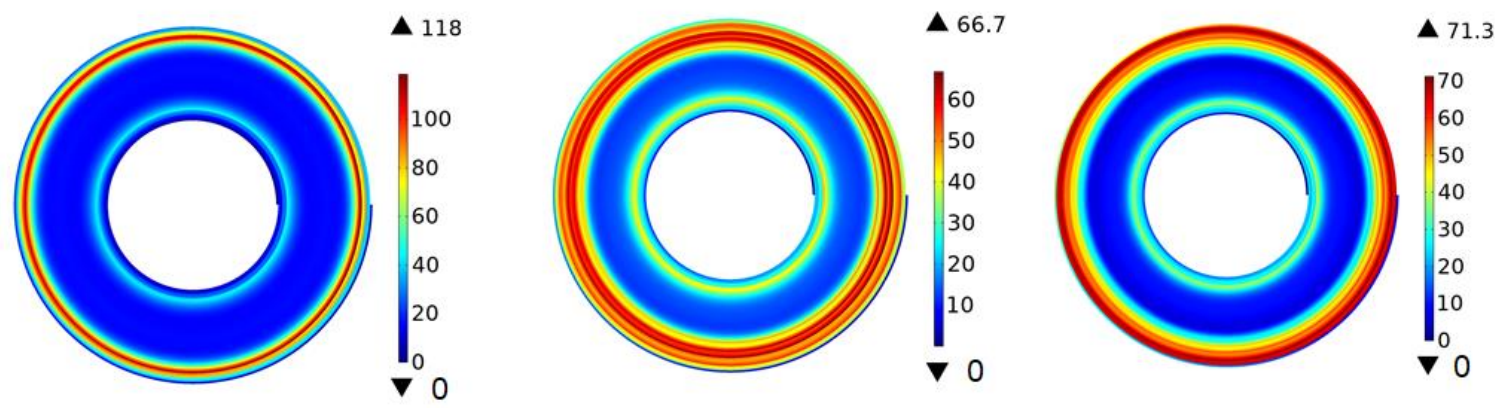

(b) Distribution of transport/azimuthal current (A)
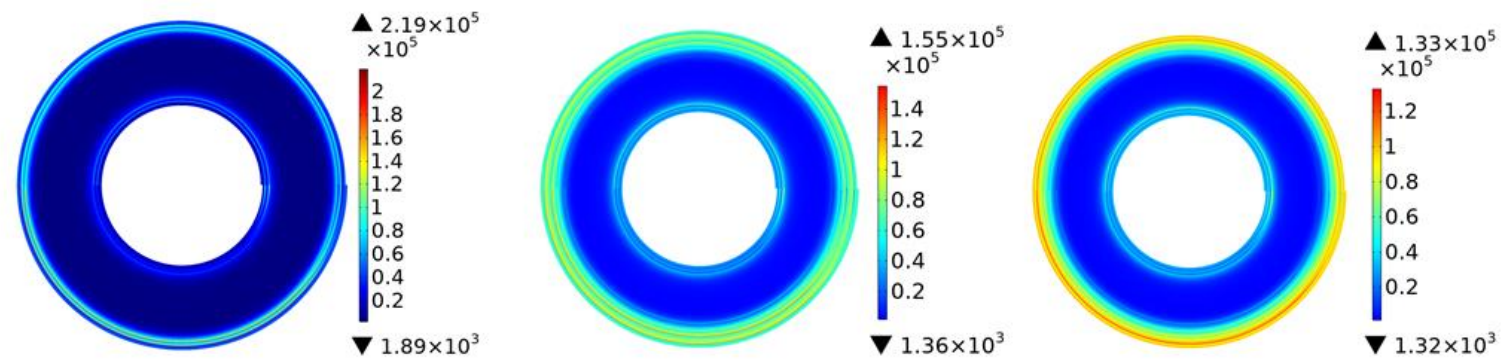

(c) Distribution of turn-to-turn loss power (W) 
Fig. 19. Case study on the grading turn-to-turn resistivity technique, (a) distribution of the grading turn-to-turn resistivity, (b) distribution of the transport(azimuthal) current induced, (c) distribution of the turn-to-turn loss power. The background field is $29 \mathrm{mT} / 50 \mathrm{~Hz}$.

\section{v. Conclusion}

To sum up, we studied the practicability of applying the NI HTS winding technique on electrical machines with high power density, which is a promising technique for electrical aircraft propulsion. The induced eddy current and loss of NI HTS coil exposed in ripple magnetic fields is analysed, which have been main warries about this technique on the machines of electrical aircraft so far. A numerical model based on circuit network method is developed for the NI HTS coil exposed to ripple background fields, ant it is validated by experiments.

Analysis using this model show that a considerable eddy current is induced in the NI HTS coil exposed to ripple fields. Most of eddy current accumulates on the outer turns of the NI HTS coil, and a very small external ripple field may induce a very high eddy current on outermost turns. This contributes to higher quench risk on the outermost turns of the NI HTS coil in machines. The induced transport current increases rapidly with the amplitude and frequency of the ripple fields, and an induced overcurrent (above the critical current) is possible when the amplitude and frequency is high enough.

A considerable AC loss is generated in the NI HTS coils, which consist of turn-to-turn loss on contacts and azimuthal loss in HTS tapes. The turn-to-turn loss is more than one order of magnitude higher than the azimuthal loss when the induced transport current is below critical current. during overcurrent operation, the azimuthal loss increases dramatically and is much more than the turn-to-turn loss. Therefore, AC loss may be challenging for the efficiency of HTS machines, when the NI coil is applied in machine environment with ripple fields, though the NI technique can significantly enhance the stability and reliability of the HTS windings.

The induced transport(azimuthal) current can be reduced significantly by increasing the turn-to-turn resistivity. Below critical current, the AC loss (turn-to-turn loss) can be significantly reduced by both decreasing the turn-toturn resistivity and increasing the turn-to-turn resistivity. Lower turn-to-turn resistivity means better thermal stability, but leads to more significant accumulation of radial current as well as turn-to-turn loss. Therefore, an optimization of turn-to-turn resistivity is required to avoid this peak point when the NI technique is applied on the HTS machines. 
A grading turn-to-turn resistivity technique is proposed for the NI HTS coil in machines, which can significantly reduce the transport current induced and meanwhile keep the thermal stability of the NI HTS coil. This considerably increases the practicability of the NI HTS technique in machines of electrical aircraft.

\section{ACKNOWLEDGMENTS}

This project was supported European Union's Horizon 2020 research and innovation programme under the Marie Sklodowska-Curie grant agreement No 799902 and by the Grant Agency VEGA under Contract 1/0151/17.

\section{References}

[1] W. Cao, B. C. Mecrow, G. J. Atkinson, J. W. Bennett, and D. J. Atkinson, "Overview of Electric Motor Technologies Used for More Electric Aircraft (MEA)," IEEE Transactions on Industrial Electronics, vol. 59, no. 9, pp. 3523-3531, 2012.

[2] $\quad$ F. Berg, J. Palmer, P. Miller, M. Husband, and G. Dodds, "HTS Electrical System for a Distributed Propulsion Aircraft," Ieee Transactions on Applied Superconductivity, vol. 25, no. 3, Jun 2015, Art. no. 5202705.

[3] B. Sarlioglu and C. T. Morris, "More Electric Aircraft: Review, Challenges, and Opportunities for Commercial Transport Aircraft," IEEE Transactions on Transportation Electrification, vol. 1, no. 1, pp. 54-64, 2015.

[4] http://www.machinedesign.com/batteriespower-supplies/future-electric-hybrid-aviation.

[5] Horizon 2020, Work Programme 2016-2017, 11, Smart, green and integrated transport.

[6] C. E. Jones, P. J. Norman, S. J. Galloway, M. J. Armstrong, and A. M. Bollman, "Comparison of Candidate Architectures for Future Distributed Propulsion Aircraft," Ieee Transactions on Applied Superconductivity, vol. 26, no. 6, Sep 2016, Art. no. 3601409.

[7] F. Berg, J. Palmer, P. Miller, and G. Dodds, "HTS System and Component Targets for a Distributed Aircraft Propulsion System," Ieee Transactions on Applied Superconductivity, vol. 27, no. 4, Jun 2017, Art. no. 3600307.

[8] X. Zhang, C. L. Bowman, T. C. O. Connell, and K. S. Haran, "Large electric machines for aircraft electric propulsion," IET Electric Power Applications, vol. 12, no. 6, pp. 767-779, 2018.

[9] I. D. Chasiotis and Y. L. Karnavas, "A Generic Multi-Criteria Design Approach Toward High Power Density and Fault-Tolerant LowSpeed PMSM for Pod Applications," IEEE Transactions on Transportation Electrification, vol. 5, no. 2, pp. 356-370, 2019.

[10] M. Zhang, F. Eastham, and W. J. Yuan, "Design and Modeling of 2G HTS Armature Winding for Electric Aircraft Propulsion Applications," Ieee Transactions on Applied Superconductivity, vol. 26, no. 3, Apr 2016, Art. no. 5205705.

[11] X. Song, Y. Wang, D. Liu, S. Wang, and D. Liang, "Short-Circuit Characteristics of a High Temperature Superconducting Wind Turbine Generator Employing a Segmented Armature Winding," IEEE Transactions on Applied Superconductivity, vol. 29, no. 5, pp. $1-5,2019$.

[12] M. Oomen, W. Herkert, D. Bayer, P. Kummeth, W. Nick, and T. Arndt, "Manufacturing and test of 2G-HTS coils for rotating machines: Challenges, conductor requirements, realization," (in English), Physica C-Superconductivity and Its Applications, Article vol. 482, pp. 111-118, Nov 2012.

[13] X. Song et al., "Ground Testing of the World's First MW-Class Direct Drive Superconducting Wind Turbine Generator," IEEE Transactions on Energy Conversion, pp. 1-1, 2019.

[14] H. H. Song, K. Gagnon, and J. Schwartz, "Quench behavior of conduction-cooled YBa2Cu3O7-delta coated conductor pancake coils stabilized with brass or copper," (in English), Superconductor Science \& Technology, Article vol. 23, no. 6, p. 10, Jun 2010, Art. no. 065021.

[15] S. Tengming, Y. Liyang, T. Daniele, and L. Pei, "High-field quench behavior and dependence of hot spot temperature on quench detection voltage threshold in a Bi 2 Sr 2 CaCu 2 O x coil," Superconductor Science and Technology, vol. 28, no. 7, p. $075014,2015$.

[16] Y. Liyang, L. Pei, S. Tengming, and S. Justin, "Quench degradation limit of multifilamentary Ag/Bi $2 \mathrm{Sr} 2 \mathrm{CaCu} 2 \mathrm{O}$ x round wires," Superconductor Science and Technology, vol. 29, no. 3, p. 035010, 2016.

[17] Y. Wang, J. Zheng, Z. Zhu, M. Zhang, and W. Yuan, "Quench behavior of high-temperature superconductor (RE)Ba2Cu3O x CORC cable," Journal of Physics D: Applied Physics, vol. 52, no. 34, p. 345303, 2019/06/24 2019.

[18] H. Song and J. Schwartz, "Stability and Quench Behavior of YBa2Cu3O7-x Coated Conductor at 4.2 K, Self-Field," Ieee Transactions on Applied Superconductivity, vol. 19, no. 5, pp. 3735-3743, Oct 2009.

[19] S. Hahn, D. K. Park, J. Bascunan, and Y. Iwasa, "HTS Pancake Coils Without Turn-to-Turn Insulation," (in English), Ieee Transactions on Applied Superconductivity, Article vol. 21, no. 3, pp. 1592-1595, Jun 2011.

[20] S. Hahn et al., "No-insulation multi-width winding technique for high temperature superconducting magnet," Applied Physics Letters, vol. 103, no. 17, Oct 212013 , Art. no. 173511.

[21] Y. Iwasa and S. Hahn, "First-cut design of an all-superconducting 100-T direct current magnet," Applied Physics Letters, vol. 103, no. 25, Dec 16 2013, Art. no. 253507. 
[22] Y. H. Choi et al., "Thermal Quench Behaviors of No-Insulation Coils Wound Using GdBCO Coated Conductor Tapes With Various Lamination Materials," (in English), Ieee Transactions on Applied Superconductivity, Article vol. 24, no. 3, p. 5, Jun 2014, Art. no. 8800105 .

[23] J.-B. Song, S. Hahn, T. Lecrevisse, J. Voccio, J. Bascunan, and Y. Iwasa, "Over-current quench test and self-protecting behavior of a 7 T/78 mm multi-width no-insulation REBCO magnet at 4.2 K," Superconductor Science \& Technology, vol. 28, no. 11, Nov 2015, Art. no. 114001 .

[24] W. D. Markiewicz, J. J. Jaroszynski, D. V. Abraimov, R. E. Joyner, and A. Khan, "Quench analysis of pancake wound REBCO coils with low resistance between turns," Superconductor Science \& Technology, vol. 29, no. 2, pp. 25001-25001, Feb 2016, Art. no. 025001 .

[25] T. M. Qu, P. C. Michael, J. Voccio, J. Bascunan, S. Hahn, and Y. Iwasa, "Persistent-current switch for pancake coils of rare earthbarium-copper-oxide high-temperature superconductor: Design and test results of a double-pancake coil operated in liquid nitrogen (77-65 K) and in solid nitrogen (60-57 K)," Applied Physics Letters, vol. 109, no. 8, Aug 2016, Art. no. 082601.

[26] W. K. Chan and J. Schwartz, "Improved stability, magnetic field preservation and recovery speed in (RE)Ba2Cu3Ox-based noinsulation magnets via a graded-resistance approach," Superconductor Science and Technology, Article vol. 30, no. 7, 2017 , Art. no. 074007.

[27] S. Hahn et al., "45.5-tesla direct-current magnetic field generated with a high-temperature superconducting magnet," Nature, 2019/06/12 2019.

[28] U. Bong et al., "A Design Study on 40 MW Synchronous Motor With No-Insulation HTS Field Winding," IEEE Transactions on Applied Superconductivity, vol. 29, no. 5, pp. 1-6, 2019.

[29] M. Furuse, M. Yoshikawa, Y. Itoh, S. Fukui, and T. Nakamura, "Fabrication and Testing of Racetrack-Shaped Double-Pancake Coil for Stator Windings of Induction-Synchronous Motor," IEEE Transactions on Applied Superconductivity, vol. 25, no. 3, pp. 1-4, 2015.

[30] P. Song, T. M. Qu, L. F. Lai, M. S. Wu, X. Y. Yu, and Z. Han, "Thermal analysis for the HTS stator consisting of HTS armature windings and an iron core for a $2.5 \mathrm{~kW}$ HTS generator," Superconductor Science and Technology, vol. 29, no. 5, p. 054007, 2016.

[31] M. Heejong, K. Yeong-Chun, P. Heui-Joo, Y. In-Keun, and P. Minwon, "An introduction to the design and fabrication progress of a megawatt class 2G HTS motor for the ship propulsion application," Superconductor Science and Technology, vol. 29, no. 3, p. 034009, 2016.

[32] M. Zhang, W. Yuan, J. Kvitkovic, and S. Pamidi, "Total AC loss study of 2G HTS coils for fully HTS machine applications," Superconductor Science \& Technology, vol. 28, no. 11, Nov 2015, Art. no. 115011.

[33] Y. Wang, H. Song, W. Yuan, Z. Jin, and Z. Hong, "Ramping turn-to-turn loss and magnetization loss of a No-Insulation (RE) Ba2Cu3Ox high temperature superconductor pancake coil," Journal of Applied Physics, vol. 121, no. 11, Mar 2017, Art. no. 113903.

[34] Y. W. Wang, M. Zhang, W. J. Yuan, Z. Y. Hong, Z. J. Jin, and H. H. Song, "Non-uniform ramping losses and thermal optimization with turn-to-turn resistivity grading in a (RE) Ba2Cu3Ox magnet consisting of multiple no-insulation pancake coils," Journal of Applied Physics, vol. 122, no. 5, Aug 2017, Art. no. 053902.

[35] Y. Wang, H. Song, D. Xu, Z. Y. Li, Z. Jin, and Z. Hong, "An equivalent circuit grid model for no-insulation HTS pancake coils," Superconductor Science \& Technology, vol. 28, no. 4, Apr 2015, Art. no. 045017.

[36] Y. Wang, C. Wan Kan, and J. Schwartz, "Self-protection mechanisms in no-insulation (RE)Ba $2 \mathrm{Cu} 3 \mathrm{O} \times$ high temperature superconductor pancake coils," Superconductor Science and Technology, vol. 29, no. 4, pp. 045007 (11 pp.)-045007 (11 pp.), April 2016.

[37] H. L. Quach et al., "Analysis on Electrical and Thermal Characteristics of a No-Insulation HTS Coil Considering Heat Generation in Steady and Transient States," IEEE Transactions on Applied Superconductivity, vol. 29, no. 5, pp. 1-6, 2019.

[38] M. Cho et al., "Combined Circuit Model to Simulate Post-Quench Behaviors of No-Insulation HTS Coil," IEEE Transactions on Applied Superconductivity, vol. 29, no. 5, pp. 1-5, 2019.

[39] S. Noguchi, R. Itoh, S. Hahn, and Y. Iwasa, "Numerical Simulation of Superconducting Coil Wound With No-Insulation NbTi Wire," Ieee Transactions on Applied Superconductivity, vol. 24, no. 3, Jun 2014, Art. no. 4900504.

[40] L. Donghui, Z. Weiwei, Y. Huadong, and Z. Youhe, "Thermal stability and mechanical behavior in no-insulation high-temperature superconducting pancake coils," Superconductor Science and Technology, vol. 31, no. 8, p. 085010, 2018.

[41] D. Liu, W. Zhang, H. Yong, and Y. Zhou, "Numerical analysis of thermal stability and mechanical response in a no-insulation hightemperature superconducting layer-wound coil," Superconductor Science and Technology, vol. 32, no. 4, p. 044001, 2019/02/15 2019.

[42] X. D. Wang et al., "Turn-to-turn contact characteristics for an equivalent circuit model of no-insulation ReBCO pancake coil," (in English), Superconductor Science \& Technology, vol. 26, no. 3, Mar 2013.

[43] Y. Wang and H. Song, "Influence of turn-to-turn resistivity and coil geometrical size on charging characteristics of no-electricalinsulation REBCO pancake coils," Superconductor Science and Technology, vol. 29, no. 7, p. 075006, 2016.

[44] M. Bonura, C. Barth, A. Joudrier, J. F. Troitino, A. Fête, and C. Senatore, "Systematic Study of the Contact Resistance Between REBCO Tapes: Pressure Dependence in the Case of No-Insulation, Metal Co-Winding and Metal-Insulation," IEEE Transactions on Applied Superconductivity, vol. 29, no. 5, pp. 1-5, 2019.

[45] M. Sohn, K. Sim, B. Eom, H. Ha, H. Kim, and K. Seong, "Controllability of the Contact Resistance of 2G HTS Coil With Metal Insulation," IEEE Transactions on Applied Superconductivity, vol. 28, no. 3, pp. 1-5, 2018.

[46] L. Jun, G. Robert, H. Ke, and H. Seungyong, "Contact resistance between two REBCO tapes under load and load cycles," Superconductor Science and Technology, vol. 30, no. 4, p. 045005, 2017.

[47] Y. Wang et al., "Analysis and Comparison Between No-Insulation and Metallic Insulation REBCO Magnet for the Engineering Design of a 1-MW DC Induction Heater," IEEE Transactions on Applied Superconductivity, vol. 27, no. 4, pp. 1-5, 2017. 